\title{
Atypical ossifying fibroma of forearm bones
}

\author{
Nareshkumar Satyanarayan Dhaniwala ${ }^{1, *}$, Mukund Naresh Dhaniwala ${ }^{2}$ \\ ${ }^{1}$ Professor, ${ }^{2}$ Resident, Professor of Orthopedics, Jawaharlal Nehru Medical College, Datta Meghe Institute of Medical Sciences \\ (Deemed University) Sawangi (Meghe), Wardha, Maharashtra, India
}

*Corresponding Author:

Email: nsdhaniwala@gmail.com

\begin{abstract}
Introduction: Ossifying fibroma is a rare tumor seen in children in diaphysis of tibia or fibula, maxilla, mandible or temporal bone. Its clinical and histological picture have variable and confusing features.

The case report hereunder describes an atypical case of ossifying fibroma involving both radius and ulna in an adolescent girl. Benign on the basis of history, the case had features of aggressive tumor on clinical and radiological examination. Histopathology did not show any features of malignancy. The patient underwent excisional biopsy followed by below elbow amputation. The patient remains asymptomatic without any features of recurrence 24 months post-surgery.
\end{abstract}

Keywords: Aty pical, Ossifying fibroma, Radius, Ulna, Aggressive.

\section{Introduction}

Ossifying fibroma of long bones, better known as osteofibrous dysplasia, is also known as campanacci disease. ${ }^{1}$ It is a rare lesion usually affecting mandible, maxilla, and temporal bone. ${ }^{2-4}$ It is rare in long bones and commonly involves tibia or fibula at diaphysis. ${ }^{1}$ It shares clinical and imaging features with several other pathologies such as fibrous dysplasia and adamantinoma. It shares histological features with other bone tumors and often cannot be distinguished without good experience in musculo skeletal pathology.

The case report herein describes an atypical case of ossifying fibroma arising from distal radius and involving ulna along with extension to volar and dorsal side in a slow painless manner. Clinically and radiologically the tumor had features of an aggressive neoplasm and was managed by amputation due to extensive soft tissue involvement.

\section{Case Report}

A 20 years old female presented to the orthopedic service of a rural tertiary care hospital with complaint of slowly progressive swelling in the distal third of left forearm for 8 years. The swelling had started spontaneously, without any history of trauma, fever or pain and had progressed gradually for initial six years. The rate of growth had increased in the last two years. There was no loss of appetite or loss of weight. The patient was managing household chores with moderate difficulty due to size of swelling and restriction of movements of forearm and wrist.

Examination revealed circumferential, hard swelling of size $12 \mathrm{~cm}$ length and $8 \mathrm{~cm}$ width at the maximum prominence in lower third of left forearm. Temperature was slightly raised and surface was smooth and lobulated. Consistency was uniformly hard except for two small areas where the feel was firm. The swelling was involving both radius and ulna bones, and it was not possible to palpate the bones in the area of swelling. The left forearm was fixed in midprone position and the wrist in neutral position. There was no neuro vascular deficit, and forearm and wrist did not have more than a jog of movement. The elbow joint was normal. Circumference of forearm was $15 \mathrm{~cm}$ more than the normal side. There was no lymphadenopathy.

X-ray of the forearm showed dense sclerotic swelling involving lower thirds of ulna and radius. The swelling was involving interosseous space and was spreading out all around the forearm. Margins were well defined with certain areas showing irregularity (Fig. 1). X-ray skull, pelvis and chest did not show any abnormality. Patient's blood investigations were within normal limits.

MRI of left forearm with wrist (Fig. 2) showed large well defined lobulated altered signal intensity lesion of size $11.5 \mathrm{~cm} \times 7.1 \mathrm{~cm}$ in the distal forearm arising from the diaphysis of distal radius and involving the distal ulna. The lesion was heterogeneously isointense on T1W1 and hyperintense on T2W2. Cortical breach was noted on the medial aspects of both the bones, with associated large soft tis sue component extending into both flexor and extensor compartment of distal forearm, abutting and displacing the tendons. Calcified soft tissue was seen extending across the wrist joint involving the flexor retinaculum. Sunburst type of periosteal reaction was seen in the distal ulna involving the interosseous membrane.

Clinico-radiologically the diagnosis was considered as Osteochondroma with malignant transformation. FNAC was done from the margin of the swelling, which did not show any malignant cells.

The patient was offered the choice of below elbow amputation in view of extensive involvement of the soft tissues, aggressive nature of the lesion practical difficulties of total tumor excision and possibility of poor post operative function. As the patient was unwilling for amputation, she underwent excisional biopsy. Per operatively, identification of nerves and 
vessels was impossible and the bony tumor tissue was found extending circumferentially and destroying deeper muscles. The radial artery got damaged during dissection. Left diaphyseal fibular graft from the patient was used to create single bone (ulna) forearm after excision of the tumor along with involved parts of the radius and ulna (Fig. 3). Post operatively the fingers, hand and the lower forearm gradually developed gangrene. The patient underwent below elbow amputation after the line of demarcation was established. The wound healed uneventfully. Excised tumor was sent for histopathological examination. Its multiple sections were studied as some of the findings were mimicking as moderately differentiated fibrosarcoma. On multiple sections it showed normal bony trabeculae at periphery without destruction or scalloping. In between the trabeculae, proliferating spindle cell lesion was visible. Spindle cells with elongated nuclei were arranged in small whorls with the nuclei showing aggressive morphology. Histological features were suggestive of os sifying fibroma (Fig. 4).

The patient remains asymptomatic 24 months after the surgery.
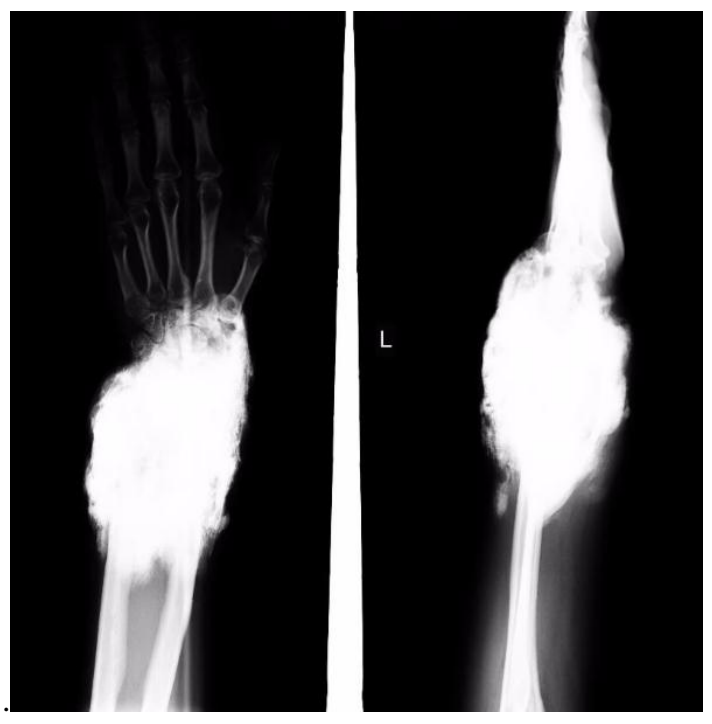

Fig. 1: X ray antero-posterior and lateral views of left forearm with wrist showing radiodense circumferential swelling involving lower third of radius, ulna and interosseous space
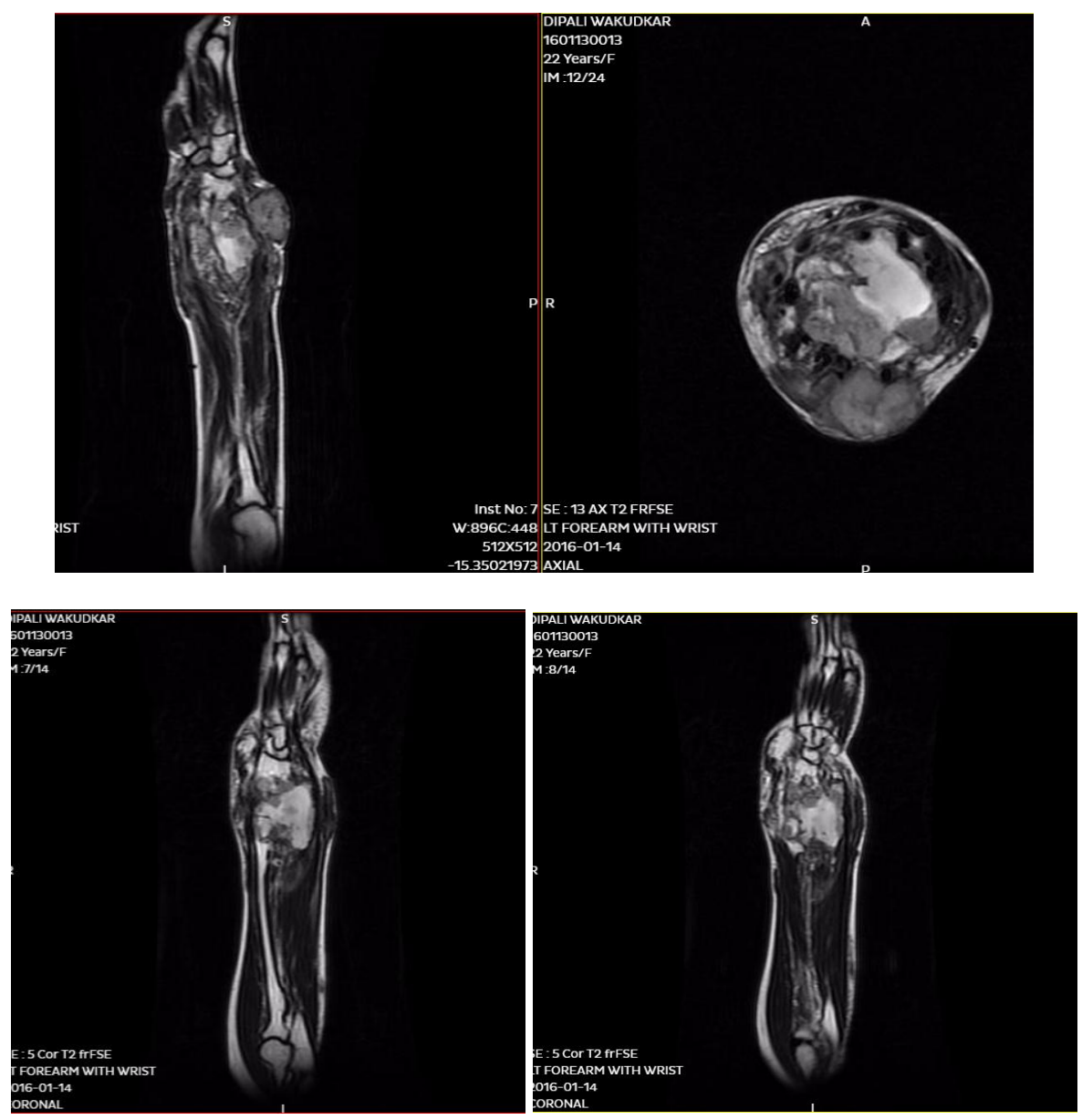

Fig. 2: MRI of forearm (sagittal, axial and coronal views) showing involvement of distal third of radius and ulna with extensive involvement of soft tissues 


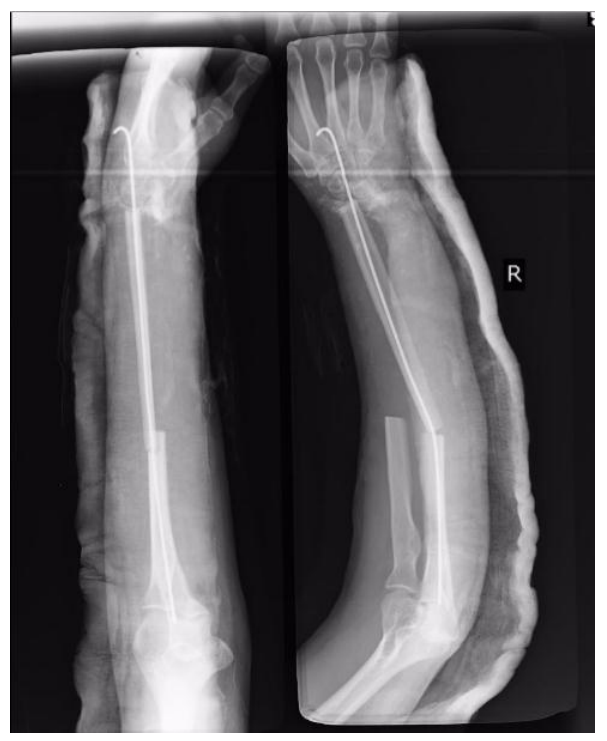

Fig. 3: $\mathrm{X}$ ray after excisional biopsy and fibular grafting

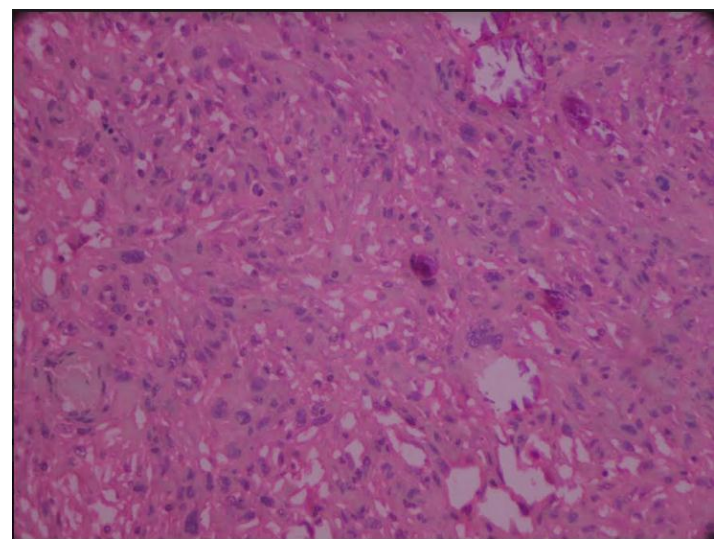

Fig. 4: Histopathology showing spindle cells with elongated nuclei arranged in whorls. No evidence of malignancy seen

\section{Discussion}

Ossifying fibroma is a rare tumor. Only about 70 cases have been the reported in the English literature. ${ }^{5}$ Long bone involvement is common in tibia and fibula. ${ }^{6}$ ${ }^{8}$ Involvement of other long bones is rare. ${ }^{9-11}$ Ulna and radius involvement is reported in isolation., ${ }^{5,12}$ Its aggressive behavior and recurrence have been noted in many studies. ${ }^{5,6}$ Regression of the tumor after fracture is also reported. ${ }^{8}$

Most commonly, the tumor occurs in children of 10 years of age or younger. Our patient started having the swelling around 14 years of age. Wang et al also reported three cases in more than 10 years age group. ${ }^{5}$ Normally, the tumor involves one bone as against involvement of both radius and ulna in the present case. Common findings on radiograph include a multilocular radiolucent lesion with sclerotic rim around the lesion. The usual mode of treatment is curettage and bone grafting. The present case is atypical in most of its features. It started at a late age and instead of remaining localized to radius, where it started first, it grew out of the bone and involved all surrounding tissues including the ulna. Its aggressive tendency was apparent on palpation on account of the variable consistency. The radiograph and MRI suggested the aggressive behavior clearly. It was not possible to treat it in the usual way due to extensive soft tissue involvement. The tumor though, aggressive was not malignant as histologically, no malignant cells were seen and the findings were suggestive of ossifying fibroma. The patient is asymptomatic at 24 months of follow up and needs regular follow up for detection of any recurrence.

\section{References}

1. Robert K. Heck Jr., Patrick c. Joy. Benign Bone Tumors \& Nonneoplastic conditions simulating Bone Tumors. Campbell's Operative Orthopedics. $13^{\text {th }}$ ed. vol 1 2017:907.

2. Cicciu M, Herford A S, Juodzbalys G, Cicciu D. Juvenile Ossifying Fibroma of the maxilla: a rare aggressive case in a young patient. J Cancer Res Ther. 2013 Apr-Jun; 9(2):324-7.

3. Nonaka CF, Pacheco DF, Nunes RP, Freitas Rde A, Miguel MC. Ossifying Fibromyxoid tumor in the mandibular gingival: case report and review of the literature: J Peridontol. 2009, Apr; 80(4):687-92.

4. Goh EK, Chok S, Lee IW, Chon KM. A case of isolated Ossifying Fibroma of the mastoid cavity of the temporal bones. Am J Otolaryngol 2006, Sep-Oct; 27(5):354-8.

5. Wang JW, Shih $\mathrm{CH}$, Chen WJ. Osteofibrous dysplasia (Ossifying Fibroma of long bones). A report of four cases and review of the literature. Clin Ortho Relat Res. 1992 May;(278):235-43.

6. Almeida Herrero F, Silvestre Munoz A, Martinoz Rodriguez M, Gomar Sancho F. Ossifying Fibroma of long bones in adults: a case report. Acta Orthop Belg 2006, Dec;72(6):786-9.

7. Szendroi M, Antal I, Arato G. Adamantinoma of long bones: a long term follow up study of 11 cases. Pathol Oncol Res, 2009 June;!5(2):209-16.

8. Mastalglia S, Mautalen C. Regression of an Ossifying Fibroma of the tibia after a fracture involving the lesion. Possible role of Peristina. Clin Cases Miner Bone Metab,2015 Sep-Oct;12(3):262-4.

9. Gopinathan NR, Prakash M, Saibaba B, Das A. Osteofirous dysplasia of clavicle clinically mimicking chronic osteomyelitis. Indian J Radiol Imaging 2016 AprJun;26(2):290-3.

10. Desprechins B, Freson M, De Boeck H, Bourgain C, Osteaux M. Ossifying Fibroma of the long bones. Radiological diagnosis, differential diagnosis and management implications. J Belge Radiol 1991 Mar;74(2):121-4.

11. Nakashima Y, Yamamuro T, Fujiwara Y, Kotoura Y, Mori E, Hamashima Y. Osteofibrous dysplasia (Ossifying Fibroma of long bones). A study of 12 cases. Cancer, 1983 Sep;52(5):909-14.

12. Goto T, Kojima T, Iijima T, Yokokura S, Kawane H, Yamamoto A, Matsuda K. Osteofibrous dysplasia of the ulna. J Orthop Sci. 2001;6(6):608-11. 